\title{
New Jersey-Wide Survey of Spotted Fever Group Rickettsia (Proteobacteria: Rickettsiaceae) in Dermacentor variabilis and Amblyomma americanum (Acari: Ixodida: Ixodidae)
}

\author{
James Occi, ${ }^{1}$ Andrea M. Egizi, ${ }^{1,2}$ Ashley Goncalves, ${ }^{1}$ and Dina M. Fonseca ${ }^{1 *}$ \\ ${ }^{1}$ Department of Entomology, Center for Vector Biology, Rutgers University, New Brunswick, New Jersey; ${ }^{2}$ Monmouth County Mosquito \\ Control Division, Tick-borne Diseases Laboratory, Tinton Falls, New Jersey
}

\begin{abstract}
For the last decade, the New Jersey $(\mathrm{NJ})$ Department of Health has reported between 42 and 144 new cases each year of "spotted fever group rickettsiosis" (SFGR), a statistic that reflects uncertainty regarding which rickettsial agents (Proteobacteria: Rickettsiaceae: Rickettsia) are infecting NJ residents. To identify the Rickettsia circulating in NJ ticks, we used a combination of conventional and real time PCR approaches to screen 560 Dermacentor variabilis Say and 245 Amblyomma americanum L. obtained from a 1-day state-wide surveillance in May 2018 and an additional 394 $D$. variabilis collected across $\mathrm{NJ}$ in 2013-2018. We found zero D. variabilis infected with Rickettsia rickettsii, the agent of Rocky Mountain spotted fever and, on average, 1.3\% infected with presumed nonpathogenic Rickettsia montanensis. We also found zero $A$. americanum infected with $R$. rickettsii, and $20 \%$ infected with Rickettsia amblyommatis, a prevalence somewhat lower than in more southern states. Overall, we conclude that it is unlikely that $R$. rickettsii vectored by $D$. variabilis is a primary cause of SFGR cases in NJ and discuss our findings in the context of known facts and current limitations. We conclude that understanding the causes of SFGR east of the Mississippi will require collaboration among medical doctors, public health authorities, and medical entomologists to follow up presumptive human cases of SFGR with detailed histories of exposure, species-specific molecular assays, and active surveillance of putative vectors and the pathogens they may carry.
\end{abstract}

\section{INTRODUCTION}

The Rickettsia (Proteobacteria: Rickettsiaceae) are Gram negative obligate intracellular bacteria often transmitted among vertebrate hosts by an arthropod vector. The species in the genus are distinguished by their pathogenicity, ecological, and biochemical profiles into four groups: the typhus group, the spotted fever group (SFG), the ancestral group, and the transitional group. ${ }^{1}$ The SFG Rickettsia include approximately 20 valid species (depending on authority ${ }^{2,3}$ ) transmitted primarily, but not exclusively, by hard ticks (Acari: Ixodida). Some of these Rickettsia are pathogenic (e.g., Rickettsia rickettsii), some are nonpathogenic (such as Rickettsia peacockii), and some may be tick commensals or symbionts (such as Rickettsia buchneri in Ixodes scapularis ${ }^{4}$ ). The complexity and diversity of SFG Rickettsia presents a challenge because serology designed to detect pathogenic species often cross-reacts with the nonpathogenic members. ${ }^{5}$

A particularly virulent tick-borne Rickettsia in the United States is $R$. rickettsii. Infection with this bacterium causes Rocky Mountain spotted fever (RMSF) and can be fatal if left untreated or if there is a delay in treatment due to misdiagnosis. ${ }^{6,7}$ Since 2000 or so, there has been a steep increase in RMSF cases presenting with reduced morbidity and mortality in the mid-Atlantic states, ${ }^{8}$ a puzzling phenomenon. Recognition of the unknown epidemiology by local Public Health authorities has, in some cases, led to a switch in the terminology from RMSF to the broader term "spotted fever group rickettsiosis" (SFGR) encompassing a range of etiologies. ${ }^{8}$

It is also unclear which vectors are transmitting SFG Rickettsia to humans in the mid-Atlantic states. Rickettsia rickettsii was first recognized as a tick-transmitted infection in the early 1900s in the Bitterroot Valley of Montana, and there the

*Address correspondence to Dina M. Fonseca, Center for Vector Biology, Rutgers University, 180 Jones Ave., New Brunswick, NJ 08901-8536. E-mail: dina.fonseca@rutgers.edu primary vector is the Rocky Mountain wood tick, Dermacentor andersoni Stiles. ${ }^{9}$ After Parker showed that Dermacentor variabilis Say was a competent vector of $R$. rickettsii, ${ }^{10}$ this tick has been considered the vector east of the Mississippi River. ${ }^{11-13}$ However, several recent studies conducted on large samples of $D$. variabilis in the eastern United States have broadly failed to find evidence of $R$. rickettsii (Table 1). Before 2010 or so, studies primarily used conventional PCR with sequencing to identify the Rickettsia found, but recent uptake of highly sensitive real-time PCR (hereafter termed qPCR) assays have also failed to detect significant rates of tick infection with $R$. rickettsii (Table 1).

The highest $R$. rickettsii infection prevalence found in $D$. variabilis from the mid-Atlantic region was $0.9 \%$, in a recent study by Kakumanu and others ${ }^{14}$ using reverse line blot to screen for multiple species of Rickettsia. Of note, in their study over $50 \%$ of the $R$. rickettsii-infected $D$. variabilis were coinfected with Rickettsia amblyommatis raising the possibility that coinfection with a more abundant Rickettsia may prevent detection of $R$. rickettsii when using standard PCR followed by sequencing. Although Rickettsia coinfection in ticks has not been reported often, ${ }^{15,16}$ it has been detected before. For example, Carmichael and Fuerst ${ }^{17}$ found one $D$. variabilis infected with R. rickettsii, Rickettsia montanensis, and Rickettsia belli. These findings underscore the need to survey for $R$. rickettsii using highly sensitive and specific assays.

Besides the right methodology, successful surveys also depend on obtaining representative samples of vectors. Whereas $D$. variabilis is considered the most likely vector, Amblyomma americanum, the lone star tick, is an increasingly common human-biter in the northeast, ${ }^{18}$ and recently, one A. americanum collected in Monmouth Co. New Jersey (NJ) was found infected with $R$. rickettsii (Table 2). Lone star ticks are competent laboratory vectors of $R$. rickettsii, ${ }^{19}$ although in field surveys, they too are rarely found infected. By far, the most frequent Rickettsia carried by lone star ticks is R. amblyommatis ( $>25 \%$, on average, Table 2). Although not a confirmed pathogen, $R$. amblyommatis has been detected in 
TABLE 1

Surveys for SFG Rickettsia in adult Dermacentor variabilis east of the Mississippi since 2000

\begin{tabular}{|c|c|c|c|c|c|c|c|}
\hline State & $\begin{array}{l}\text { Number } \\
\text { tested }\end{array}$ & $\begin{array}{l}\text { Rickettsia } \\
\text { rickettsii }\end{array}$ & $\begin{array}{c}\text { Rickettsia } \\
\text { amblyommatis }\end{array}$ & $\begin{array}{c}\text { Rickettsia } \\
\text { parkeri }\end{array}$ & $\begin{array}{c}\text { Rickettsia } \\
\text { montanensis }\end{array}$ & $\begin{array}{c}\text { Total \% SFG } \\
\text { Rickettsia }\end{array}$ & Reference \\
\hline MD & 392 & 0 & 0 & 0 & 3.8 & 6.1 & 41 \\
\hline $\mathrm{TN}$ & 548 & 0 & 2.5 & NT & 10 & 15 & 42 \\
\hline KY & 179 & 0 & 1.1 & 0.5 & 4.4 & 5 & 43 \\
\hline US & 1,400 & 0.07 & 0.1 & 0 & 3.8 & 4 & $37^{*}$ \\
\hline VA & $522 \dagger$ & 0 & 0 & 0 & 1.7 & 1.7 & 44 \\
\hline VA & 2,276 & 0 & 0.04 & 0.75 & 0.18 & 0.96 & 45 \\
\hline $\mathrm{KY}, \mathrm{TN}$ & 299 & 0 & 0 & 0 & 3.3 & 3.3 & 46 \\
\hline US & 4,792 & NT & NT & NT & 2.8 & 2.8 & $47 \ddagger$ \\
\hline TN & 2,515 & 0 & 0 & 0 & 1.2 & 3.6 & $48 \S$ \\
\hline $\mathrm{NC}$ & 532 & 0.9 & 11.8 & 0.6 & 2 & $54.7 \|$ & $14 \|$ \\
\hline 12 states & 284 & 0 & 3.5 & 0 & 2.1 & 5.3 & $49^{\star}$ \\
\hline Mean\# & - & $0.10 \pm 0.55$ & $1.90 \pm 7.24$ & $0.21 \pm 0.62$ & $3.21 \pm 5.06$ & - & - \\
\hline
\end{tabular}

NT = not tested; SFG = spotted fever group. For each Rickettsia species, numbers in columns represent per cent prevalence. Rickettsia other than those listed are included under Total \% SFG Rickettsia. We only included studies with a minimum of 100 field-collected ticks and excluded studies focusing on nonhuman host-associated ticks.

* Used the data from mid-Atlantic states only.

†Tested in 153 pools.

$\ddagger$ Only looked for $R$. montanensis.

§Only sequenced 31 .

UThis includes all Rickettsia (SFG and typhus group as well as several candidate Rickettsia).

- Prevalence of each Rickettsia species was calculated based on total number of ticks tested.

\# Means $\pm 1.96 \times$ SD $(95 \% \mathrm{Cl})$.

sera of presumed SFGR patients. ${ }^{20}$ In fact, it was proposed that exposure to $R$. amblyommatis through an $A$. americanum bite could be contributing to the number of reported cases of spotted fever group rickettsioses in the mid-Atlantic states. ${ }^{8}$ Amblyomma americanum is also capable of carrying Rickettsia parkeri, ${ }^{21}$ a pathogen that primarily infects the Gulf Coast tick, Amblyomma maculatum, a southern tick species that is expanding north but is still undetected in $\mathrm{NJ}^{22-24}$

Discussions about the etiology of SFGR in NJ with members of the NJ Department of Health and CDC prompted us to survey Rickettsia in putative NJ hard tick vectors and compare their occurrence and distribution to that of reported human cases of SFGR in NJ. To sample $D$. variabilis and $A$. americanum from across NJ, we developed the "2018 NJ Tick Blitz," an experiment in operational outreach ${ }^{25}$ that provided us with specimens from all $21 \mathrm{NJ}$ counties collected on the same day almost simultaneously.

Specifically, in this study, we 1) reviewed representative surveys of Rickettsia in D. variabilis and in A. americanum populations east of the Mississippi, 2) surveyed specimens of $D$. variabilis and $A$. americanum from across NJ for Rickettsia using the most sensitive methodology available to us, 3) compared prevalence of different Rickettsia in ticks and reported human SFGR cases at the county level, and, in addition, 4) evaluated the costs and benefits of testing pools versus individual ticks to potentially reduce the cost of testing for Rickettsia in ticks. Overall, our aim was to contribute to the understanding of the causes of SFGR in the eastern United States and to the development of better preventive and management approaches.

\section{MATERIALS AND METHODS}

We reviewed the literature on SFGR surveys in Dermacentor variabilis and $A$. americanum and present them in tabular form (Tables 1 and 2). We focused on studies that used highly sensitive and species-specific methodologies to identify the

TABLE 2

Surveys from the literature for SFG Rickettsia in Amblyomma americanum in locations east of the Mississippi

\begin{tabular}{|c|c|c|c|c|c|c|}
\hline State & Number tested & $\begin{array}{c}\text { Rickettsia } \\
\text { rickettsii }\end{array}$ & $\begin{array}{c}\text { Rickettsia } \\
\text { montanensis }\end{array}$ & $\begin{array}{c}\text { Rickettsia } \\
\text { parkeri }\end{array}$ & $\begin{array}{c}\text { Rickettsia } \\
\text { amblyommatis }\end{array}$ & Reference \\
\hline TN & $655^{\star}$ & 0 & 0.3 & 0 & 40 & 42 \\
\hline KY & 108 & NT & 0 & 0 & 27.8 & 43 \\
\hline \multirow[t]{2}{*}{ VA } & 1,969 (nymphs) & 0 & NT & 0 & 55.9 & 50 \\
\hline & 576 (adults) & 0 & NT & 0 & 72.8 & \\
\hline NC & 459 & 0.2 & 0 & 2.1 & 90.9 & 51 \\
\hline \multirow[t]{2}{*}{ VA } & $2,010-2,509 \dagger$ & 0 & 0 & 0 & 26.9 & 44 \\
\hline & $2,011-252 \dagger$ & 0 & 0 & 0 & 54.9 & \\
\hline GA & 526 & 0 & 0.4 & 0.47 & 27.4 & 52 \\
\hline \multirow[t]{3}{*}{ VA†† } & 225 (larvae) & NT & NT & NT & $7 \S$ & 53 \\
\hline & 34 (nymphs) & NT & NT & NT & 47 & \\
\hline & 81 (adults) & NT & NT & NT & 42 & \\
\hline FL & $160^{\star}$ & 0 & 0 & 0 & 33 & 54 \\
\hline $\mathrm{FL}$ & $1,312 \dagger$ & 0 & 0 & 0.16 & 29 & 55 \\
\hline NJ & 1,858 (nymphs) & 0.05 & 0 & 0.00 & 25 & 22 \\
\hline Mean\| & - & $0.025 \pm 0.12$ & $0.08 \pm 0.31$ & $0.25 \pm 1.24$ & $44.05 \pm 39.43$ & - \\
\hline
\end{tabular}

Unless otherwise noted, number tested refers to adults tested, and values for each Rickettsia species reflect \% positive. We only included studies with a minimum of 100 ticks and excluded studies focusing on nonhuman host-associated ticks.

* Nymphs and adults tested.

$\dagger$ All life stages tested.

‡ Larvae tested in pools of 15 (infection prevalence assumes one positive tick per pool).

§ Prevalence in larvae not included in calculation of mean.

$\|$ Means $\pm 1.96 \times \mathrm{SD}(=95 \% \mathrm{Cl})$. 
Rickettsia detected and, therefore, limited our summary to peer-reviewed literature published on or after 2010 primarily because many of the earlier studies used methodologies that were not species specific, such as immunoassays. We focused on surveys conducted east of the Mississippi because they are the most relevant comparisons to our findings in NJ. In addition, we only incorporated studies that tested more than 100 ticks because previous studies have shown that $R$. rickettsii infection rates in ticks are expected to be low ( 0-5\%) (Table 1), and sufficient sample sizes are needed for reliable estimates of prevalence. We excluded studies where $D$. variabilis and $A$. americanum were collected from nonhuman hosts because some hosts can be reservoirs for Rickettsia, and this could bias the estimates of infection prevalence.

Tick collections. During the 2018 NJ Tick Blitz, ticks were collected across all $21 \mathrm{NJ}$ counties by sweeping the vegetation along $300-\mathrm{m}$ transects during the morning of May 10 , $2018 .^{25}$ Ecotonal areas were chosen to target $D$. variabilis, including open grassland or meadows adjoining forest or woodland, prime habitat for the meadow vole (Microtus pennsylvanicus), ${ }^{26}$ the preferred host of larval and nymphal $D$. variabilis. ${ }^{27}$ As described, ${ }^{25}$ to simplify the process for firsttime tick collectors, we provided volunteers from each county mosquito control program with a lightweight collapsible "tick sweep" made of $0.25 \mathrm{~m}^{2}$ crib flannel (buybuy Baby, Union Township, NJ) with a PVC pipe handle. Participants removed ticks from the sweep with masking tape and placed the tape inside plastic Ziploc (SC Johnson, Racine, WI) bags with a small piece of wet paper towel to keep ticks alive until they were brought to the Rutgers Center for Vector Biology by a courier later that week. In the laboratory, ticks were removed from the tape with flamed forceps, identified and placed in $80 \%$ ethanol, and stored at $4^{\circ} \mathrm{C}$ before DNA extraction. From Tick Blitz collections, we obtained 560 D. variabilis and 245 A. americanum for analysis. To contrast this approach (onetime collection by multiple individuals), we also examined ticks collected over multiple time points by a single individual during 2013-2018 across the state of $N J(N=394 D$. variabilis collected). Ticks were flagged opportunistically from ecotonal areas such as power and pipeline rights of way, paths, and small roads adjacent to forests and public parks. Because these were somewhat random surveys, we did not establish transects. Instead, we surveyed for a minimum of 15 minutes at each site and collected information on length of survey, date, weather conditions, and GPS coordinates as well as took photographs of each site. In all cases, we identified ticks to species using standard taxonomic keys. ${ }^{28,29}$

DNA isolation and spotted fever group Rickettsia testing. DNA isolation of Tick Blitz specimens. We removed and preserved $D$. variabilis and $A$. americanum collected during the Tick Blitz from their ethanol-filled vials, allowed them to air dry, placed individual ticks in single wells of 96-well plates, and extracted tick DNA with Qiagen DNeasy 96 Blood and Tissue Kits (Qiagen, Germantown, MD) in batches of 188, following the manufacturer's instructions, with one extraction control and one empty well per plate that we later used as a PCR-negative control. The ticks were first homogenized with a Qiagen TissueLyser bead mill (Qiagen) using 5-mm stainless steel balls (OPS Diagnostics, Lebanon, NJ). We eluted DNA from each column in $160 \mu \mathrm{L}$ of Qiagen elution buffer AE for adults and $80 \mu \mathrm{L}$ for nymphs in two separate elutions.

DNA isolation from 2013 to 2018 field surveys. We removed preserved ticks one by one from their storage vials, rinsed them clean with fresh $70 \%$ ethanol, and allowed them to air dry. We extracted DNA from individual ticks using DNeasy Blood and Tissue single column kits (Qiagen) following the manufacturer's instructions, after they were macerated inside microfuge tubes with Qiagen tissue lysis buffer ATL using sterile tuberculin needles (Becton Dickinson, Franklin Lakes, $\mathrm{NJ})$. We eluted DNA from each column in $160 \mu \mathrm{L}$ of Qiagen elution buffer $A E$ for adult ticks and $80 \mu \mathrm{L}$ for nymphs.

qPCR testing of Tick Blitz specimens. Because we expected a low prevalence of SFG Rickettsia in D. variabilis $(<5 \%$, Table 1), we created tick DNA pools by taking $20 \mu \mathrm{L}$ of eluted DNA from each of the eight wells in each column of a 96-well extraction plate and combined them into a single tube (one 96well plate $=12$ pools of 7-8 ticks). We screened pools of D. variabilis for SFG Rickettsia with a TaqMan qPCR assay targeting the $17-\mathrm{kD}$ antigen ${ }^{30}$ but using an minor groove binding (MGB) quencher instead of TAMRA (Table 3) using the TaqMan Environmental Master Mix 2.0 (Applied Biosystems,

TABLE 3

Primers and probes used for amplification and sequencing of SFG Rickettsia from Dermacentor variabilis and Amblyomma americanum

\begin{tabular}{|c|c|c|c|c|}
\hline Organism & Primer/probe & Target & Sequence $\left(5^{\prime}-3^{\prime}\right)$ & Reference \\
\hline SFG Rickettsia & $\begin{array}{l}\text { Pfmt-F2 } \\
\text { Pspacer-R1 }\end{array}$ & $n-f-m e t-23 s-r R N A$ & $\begin{array}{l}\text { AAGAGAGTAAAAAAGCTTTG } \\
\text { TGGGTTTGCCACATATAGC }\end{array}$ & This study \\
\hline SFG Rickettsia & $\begin{array}{l}\text { Pglta-1F } \\
\text { Pglta-2R }\end{array}$ & gltA & $\begin{array}{l}\text { ATTGCTTTACTTACGACCC } \\
\text { TTCAAGTTCTATTGCTATTTG }\end{array}$ & This study \\
\hline SFG Rickettsia & $\begin{array}{l}\text { R190-70p } \\
\text { Rr190.602n }\end{array}$ & ompA & $\begin{array}{l}\text { ATGGCGAATATTTCTCCAAAA } \\
\text { AGTGCAGCATTCGCTCCСCCT }\end{array}$ & Ref. 31 and 56 \\
\hline SFG Rickettsia & $\begin{array}{l}\text { R17K128F2 } \\
\text { R17K238 R } \\
\text { R17K202 Probe }\end{array}$ & $17 \mathrm{kD}$ & $\begin{array}{l}\text { GGGCGGTATGAAYAAACAAG } \\
\text { CCTACACCTACTCCVACAAG } \\
\text { FAM-CCGAATTGAGAACCAA } \\
\text { GTAATGC-MGB }\end{array}$ & 30 \\
\hline Rickettsia amblyommatis & $\begin{array}{l}\text { Ra477 F } \\
\text { Ra618 R-mod } \\
\text { Ra532 Probe-mod }\end{array}$ & ompB & $\begin{array}{l}\text { GGTGCTGCGGCTTCTACATTAG } \\
\text { CCATTAGTAACATTTAATGTACCG } \\
\text { TTAACAC } \\
\text { VIC-CACTTGGACAGAATGCTT-MGB }\end{array}$ & Ref. 57 and 22 \\
\hline Rickettsia rickettsii & $\begin{array}{l}\text { RR1370F } \\
\text { RR1494R } \\
\text { RR1425B Probe }\end{array}$ & ompB & $\begin{array}{l}\text { ATAACCCAAGACTCAAACTITGGTA } \\
\text { GCAGTGTTACCGGGATTGCT } \\
\text { FAM-TTAAAGTTCCTAATGCTATA } \\
\text { ACCCTTACC-MGB }\end{array}$ & Ref. 58,50 , and his study \\
\hline
\end{tabular}


Forest City, CA). Amplification conditions were an initial incubation of $95^{\circ} \mathrm{C}$ for 10 minutes followed by 40 cycles of $95^{\circ} \mathrm{C}$ for 15 seconds and $60^{\circ} \mathrm{C}$ for 60 seconds in an Applied Biosystems 7,500 qPCR machine. If the pool tested negative, we took no further action. If the pool was positive, we returned to the original extraction plate and tested the seven or eight individual tick extracts with the 17-kD assay. Positive ticks were also tested with a TaqMan primer/probe system specific for R. rickettsii outer membrane protein B (ompB) (Table 3). We used serial dilutions of a synthetic DNA fragment matching the $R$. rickettsii ompB target region as the positive control. Tick extracts that were positive with the 17-kD assay and negative for $R$. rickettsii ompB were amplified with regular PCR primers for ompA (532-bp product) using the conditions described by Regnery and others ${ }^{31}$ as well as primers designed for this study targeting citrate synthase gltA (906 bp product, Table 3). PCR products $(5 \mu \mathrm{L})$ were electrophoresed in $1 \%$ agarose (Sigma-Aldrich, St. Louis, MO) in 1X TAE (40 mM Tris base, $20 \mathrm{mM}$ acetic acid, and $1 \mathrm{mM}$ EDTA). The remaining PCR product was treated with ExoSAP-IT (Applied Biosystems, Waltham, MA) to enzymatically digest free primers or nucleotides. The purified template was premixed with each of the primers separately and sent to Genscript (Piscataway, NJ) for sequencing. When possible, a consensus sequence of the positive and negative strands was generated using Sequencher DNA analysis software (v. 3.5, Gene Codes Corporation, Ann Arbor, MI) and entered into NCBI BLAST (https://blast.ncbi.nlm.nih.gov/Blast.cgi) to identify the sequences to species level. If one of the primers did not generate a clean sequence, we blasted using only the other strand.

We screened individual $A$. americanum with a qPCR duplex targeting 1) the $17 \mathrm{kD}$ of all SFG Rickettsia (the same used for the $D$. variabilis testing) as well as 2) an $R$. amblyommatis outer membrane protein B (ompB) (Table 3). As in Egizi and others, ${ }^{22}$ primer and probe sequences ending in "mod" were shortened to accommodate the switch to an MGB quencher (Table 3). Reactions $(20 \mu \mathrm{L})$ were denatured at $95^{\circ} \mathrm{C}$ for 10 minutes followed by 40 cycles of $95^{\circ} \mathrm{C}$ for 15 seconds and $60^{\circ} \mathrm{C}$ for 1 minute. Positive controls were synthesized dsDNA fragments matching the target qPCR regions (GeneStrings, Invitrogen, Carlsbad, CA).

To rule out the possibility that $R$. amblyommatis-infected ticks were coinfected with $R$. rickettsii, we tested all $17-\mathrm{kD}$ and $R$. amblyommatis-positive $A$. americanum with the $R$. rickettsii ompB qPCR assay (Table 3). Reaction volumes, conditions, and controls were the same as for the $D$. variabilis screening. Because recently Egizi and others ${ }^{25}$ tested more than 1,800 NJ $A$. americanum using a specific qPCR assay for $R$. parkeri and failed to detect this Rickettsia, which is unreported in $\mathrm{NJ}$, we chose to forego using this test on our specimens.

PCR testing of ticks from 2013 to 2018 field surveys. To test this group of specimens, we used primers for the region spanning the rickettsial methionyl-tRNA formyl transferase (fmt) and 23s ribosomal RNA locus. We designed the primers (Table 3) to match a broad range of Rickettsia using an alignment of 13 publicly available SFG Rickettsia species known to occur in the northeastern United States (Online Supplemental Information, Supplemental Table S1). We optimized and validated the primers using purified Rickettsia canadensis genomic DNA (ATCC VR-1444, American Type Culture Collection, Manassas, VA), $D$. variabilis infected with $R$. montanensis, and $A$. americanum infected with $R$. amblyommatis. The primers
Pfmt-2F and PSpacer-1R amplified an 860-bp PCR product. Cycle conditions were an initial 10 minutes at $95^{\circ} \mathrm{C}$, then 35 cycles of $93^{\circ} \mathrm{C}$ for 30 seconds, $50^{\circ} \mathrm{C}$ for 3 seconds, and $68^{\circ} \mathrm{C}$ for 15 seconds followed by a single cycle of $72^{\circ} \mathrm{C}$ for 10 minutes as instructed by the ThermoFisher AmpliTaq Gold Fast PCR Mix (Applied Biosystems) kit. Five microliters of PCR products was electrophoresed in 1\% agarose (Sigma-Aldrich) in 1X TAE (40 mM Tris base, $20 \mathrm{mM}$ acetic acid, and $1 \mathrm{mM}$ EDTA). We visualized, purified, sequenced, and analyzed the PCR products as described previously.

Ticks positive for Rickettsia were also examined with SFGspecific citrate synthase ( $g / t A)$ primers and the product sequenced (Table 3 ). We designed the gltA primers to amplify an 896-bp fragment in all commonly encountered SFG Rickettsia in the northeastern United States. We used $R$. canadensispurified DNA, $R$. amblyommatis-infected $A$. americanum, and $R$. montanensis-infected $D$. variabilis as positive controls. The amplification followed manufacturer's protocol (AmpliTaq, Applied Biosystems), with an initial heating of $94^{\circ} \mathrm{C}$ for 10 minutes, followed by 35 cycles of $94^{\circ} \mathrm{C}$ for 45 seconds (denature), $50^{\circ} \mathrm{C}$ for 45 seconds (annealing), and extension at $50^{\circ} \mathrm{C}$ for 45 seconds. We visualized, purified, sequenced, and analyzed the PCR products as described previously.

\section{RESULTS}

From the Tick Blitz, we obtained adult $D$. variabilis from each of the $21 \mathrm{NJ}$ counties (mean number per county $=25.9, \mathrm{SD}=$ 35.9), tested a total of 560 specimens in 70 pools, and failed to detect Rickettsia rickettsii. We also tested 394 D. variabilis specimens from the 2013 to 2018 field surveys with conventional PCR and, again, failed to detect $R$. rickettsii. Instead, from both datasets, we identified $D$. variabilis infected with $R$. montanensis (eight from the Tick Blitz and four from the 2013 to 2018 specimens), resulting in an infection prevalence with $R$. montanensis of $1.4 \%$ (8/560) and $1.0 \%$ (4/394), for specimens from the Tick Blitz and 2013-2018 surveys, respectively, which are not statistically different $(x 2=0.31, P=$ 0.57 ). Therefore, we combined the datasets and obtained an overall prevalence of $R$. montanensis in NJ D. variabilis of $1.3 \%$ (12/954, Table 4, Figure 1) that matches those in other midAtlantic populations (Table 1).

Sequences of the methionyl-tRNA-23s region from Rickettsia-positive $D$. variabilis had a $100 \%$ match to multiple strains of $R$. montanensis in GenBank, sequences of ompA had a match of $98.9 \%$, and the gltA match was $99.5 \%$. In all cases, the query coverage was $100 \%$. Furthermore, when we tested ticks from positive pools of $D$. variabilis individually, only a single tick in each pool was positive. Because minimum infection rate calculations assume a single positive specimen per pool, ${ }^{32}$ we found that pooling did not alter the estimated infection rate.

We tested $245 A$. americanum (85 adults and 160 nymphs all collected during the same day in May 2018). ${ }^{25}$ We did not detect $R$. rickettsii, but overall $20 \%$ of the $A$. americanum were positive for $R$. amblyommatis (49 positives, 22 adults, and 27 nymphs). This prevalence is relatively low, but still within the $95 \% \mathrm{Cl}$ for $R$. amblyommatis prevalence in $A$. americanum reported by others (Table 2$)$. Most (91.3\%) of the lone star ticks tested were from southern $\mathrm{NJ}$ counties, and interestingly, the few collected from the two northernmost counties within the current known range of $A$. americanum in $\mathrm{NJ}$ (Middlesex and 
TABLE 4

New cases of SFGR reported to NJ DOH by each NJ county in $2018^{*}$, average incidence ( \pm SD) from 2010 to 2018 (per 100,000 residents) and prevalence of SFG Rickettsia-positive Dermacentor variabilis and SFG Rickettsia-positive Amblyomma americanum in New Jersey

\begin{tabular}{|c|c|c|c|c|c|c|c|c|}
\hline \multirow[b]{2}{*}{ County } & \multirow[b]{2}{*}{ SFGR } & \multirow[b]{2}{*}{$\begin{array}{c}\text { Average } \\
\text { incidence } \\
\pm S D\end{array}$} & \multicolumn{3}{|c|}{ Dermacentor variabilis } & \multicolumn{3}{|c|}{ Amblyomma americanum } \\
\hline & & & $\begin{array}{l}\text { Number } \\
\text { tested }\end{array}$ & $\begin{array}{l}\text { Number } \\
\text { positive }\end{array}$ & $\begin{array}{c}\% \\
\text { Ticks } \\
\text { positive }\end{array}$ & $\begin{array}{l}\text { Number } \\
\text { tested }\end{array}$ & $\begin{array}{l}\text { Number } \\
\text { positive }\end{array}$ & $\begin{array}{c}\% \\
\text { Ticks } \\
\text { positive }\end{array}$ \\
\hline Atlantic & 14 & $4.2 \pm 3.0$ & 16 & 1 & 6.2 & 25 & 3 & 12.0 \\
\hline Bergen & 6 & $0.3 \pm 0.2$ & 35 & 1 & 2.8 & NA & & \\
\hline Burlington & 7 & $1.4 \pm 0.9$ & 6 & 1 & 16.6 & 39 & 3 & 7.7 \\
\hline Camden & 4 & $0.9 \pm 0.6$ & 5 & 0 & 0 & 17 & 2 & 12.0 \\
\hline Cape May & 1 & $2.3 \pm 2.0$ & 5 & 0 & 0 & 3 & 0 & 0 \\
\hline Cumberland & 4 & $3.1 \pm 2.4$ & 4 & 0 & 0 & 30 & 2 & 6.7 \\
\hline Essex & 4 & $0.4 \pm 0.2$ & 44 & 1 & 2.3 & NA & & \\
\hline Gloucester & 12 & $3.1 \pm 2.0$ & 8 & 1 & 12.5 & 44 & 5 & 11.4 \\
\hline Hudson & 1 & $0.2 \pm 0.2$ & 87 & 1 & 1.1 & NA & & \\
\hline Hunterdon & 7 & $4.7 \pm 4.3$ & 26 & 0 & 0 & NA & & \\
\hline Mercer & 3 & $0.4 \pm 0.3$ & 24 & 1 & 4.2 & NA & & \\
\hline Middlesex & 6 & $0.5 \pm 0.3$ & 50 & 0 & 0 & 16 & 0 & 0 \\
\hline Monmouth & 27 & $1.3 \pm 1.3$ & 153 & 2 & 1.3 & 2 & 1 & 50.0 \\
\hline Morris & 3 & $0.4 \pm 0.3$ & 119 & 0 & 0 & NA & & \\
\hline Ocean & 27 & $2.2 \pm 1.3$ & 178 & 2 & 1.1 & 65 & 32 & 49.2 \\
\hline Passaic & 0 & $0.4 \pm 0.3$ & 13 & 0 & 0 & NA & & \\
\hline Salem & 3 & $3.6 \pm 2.2$ & 13 & 0 & 0 & 3 & 1 & 33.3 \\
\hline Somerset & 4 & $0.9 \pm 0.7$ & 54 & 0 & 0 & 1 & 0 & 0 \\
\hline Sussex & 3 & $0.7 \pm 0.8$ & 27 & 1 & 2.7 & NA & & \\
\hline Union & 6 & $0.6 \pm 0.3$ & 65 & 0 & 0 & NA & & \\
\hline Warren & 2 & $0.8 \pm 1.0$ & 22 & 0 & 0 & NA & & \\
\hline Total & 144 & $1.5 \pm 2.0$ & 954 & 12 & & 245 & 49 & \\
\hline
\end{tabular}

Somerset) ${ }^{25}$ were negative for $R$. amblyommatis (Figure 1). All A. americanum positive with the 17-kD qPCR assay were also positive with the $R$. amblyommatis ompB qPCR assay. We amplified and sequenced the ompA locus of SFG Rickettsia from two positive $A$. americanum and confirmed by sequencing that $R$. amblyommatis was the Rickettsia present.

\section{DISCUSSION}

We performed a representative survey across all $21 \mathrm{NJ}$ counties of SFG Rickettsia in two tick species that have been considered critical Rickettsia vectors: the American dog tick, $D$. variabilis, and the lone star tick, $A$. americanum, and failed to detect $R$. rickettsii. Instead, we detected $R$. montanensis in $D$. variabilis and $R$. amblyommatis in $A$. americanum. Like $R$. amblyommatis, $R$. montanensis was until recently considered nonpathogenic to humans, but there has been at least one case report associating this Rickettsia with human illness. ${ }^{33}$ Overall, our results echo a growing chorus of findings that $R$. rickettsii is rare in ticks east of the Mississippi River.

To assess the effect of methodology, for $D$. variabilis, we compared two different collecting approaches (single collector over time versus simultaneous collections by multiple people), two strategies to detect and identify Rickettsia (sequencing of two loci versus Rickettsia specific qPCR), and testing pooled versus individual ticks. Overall, we found similar rates of infection by the same Rickettsia species irrespective of the methodology, indicating that access to qPCR technology was not mandatory for relevant assessment of Rickettsia prevalence. However, qPCR allowed us to pool samples. When a pool of $D$. variabilis was positive for Rickettsia, we invariably found that only a single tick was positive in that pool indicating this methodology afforded us considerable savings in reagents and time with no loss of information. Agencies in NJ wanting to detect Rickettsia in $D$. variabilis on a limited budget might consider pooling.

Because $A$. americanum is expanding across $N J$, we expected to find some heterogeneity in patterns of association with Rickettsia. However, the rates of prevalence of $R$. amblyommatis detected fell within the published range, and although we remarked that specimens from the two northernmost counties were not infected with $R$. amblyommatis, the numbers of ticks tested there were understandably relatively low.

Although SFG Rickettsia are an emerging group of bacteria with new species discovered and identified each year, ${ }^{34}$ our strategy of using a set of PCR primers (fmt-23S) and a qPCR assay (17 kD) optimized for "all SFG Rickettsia," would have detected both known and potentially undescribed SFG Rickettsia species. In addition, we are confident that our approach did not miss $R$. rickettsii in coinfections due to the use of a highly sensitive qPCR targeting this species. ${ }^{14}$

The question remains, what is causing human cases of SFGR in NJ (e.g., 144 cases in 2018, Table 4)? The currently available diagnostic tests for human infection with $R$. rickettsii do not differentiate among SFG Rickettsia: in fact, both $R$. montanensis and $R$. amblyommatis will cross-react with $R$. rickettsii antibodies in human serological tests causing a misdiagnosis of RMSF. ${ }^{33,35,36}$ While others have proposed that $R$. amblyommatis from a bite by $A$. americanum may result in a positive SFGR case, ${ }^{37}$ this hypothesis does not explain the SFGR cases in the northern $\mathrm{NJ}$ counties where $A$. americanum is presumed absent (Figure 1). However, standardized active or passive tick surveillance is currently nonexistent in $\mathrm{NJ}$ outside of Monmouth Co., ${ }^{25}$ so it is possible that the 

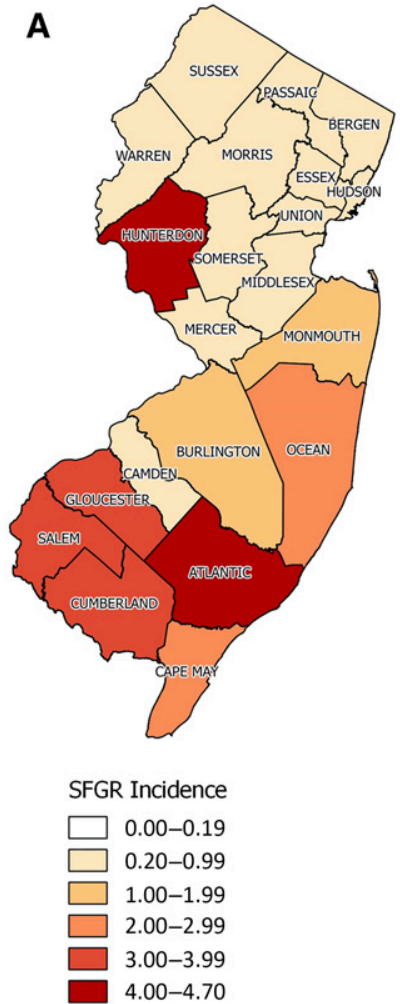

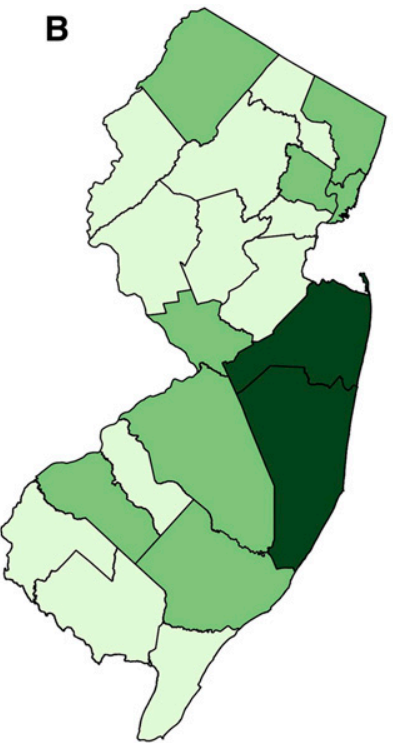

R. montanensis positive ticks

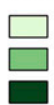

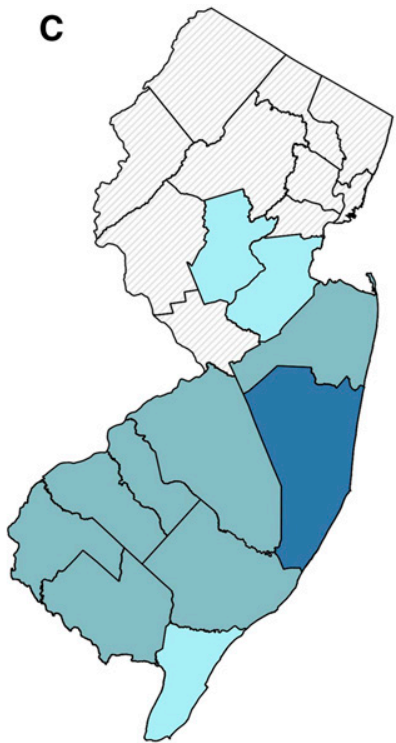

R. amblyommatis positive ticks

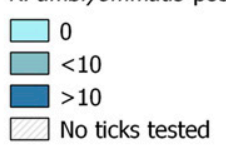

Figure 1. Map of New Jersey showing county-level (A) average incidence (\# per 100,000 residents) of human cases of spotted fever group rickettsiosis; (B) number of Dermacentor variabilis positive for Rickettsia montanensis and (C) number of Amblyomma americanum positive for Rickettsia amblyommatis. Refer to Table 4 for detailed information on number of ticks tested in each county and percent of infected ticks. This figure appears in color at www.ajtmh.org.

distribution of $A$. americanum in NJ has expanded farther north than known, undetected.

A standardized tick and tick-borne disease surveillance program in NJ (and across the northeast) is needed to investigate the growing tide of tick-borne diseases afflicting the millions living in this region. ${ }^{38,39}$ We propose that the best steps forward toward understanding the causal factors underlying the significant numbers of SFGR cases in NJ involve collaborative teams of physicians, medical entomologists, diagnostic laboratories, and public health professionals to positively identify the vectors, reservoirs, and pathogen(s) associated with local SFGR cases. Although some may argue that additional costs for specific identification are not justified because treatment is the same for all Rickettsia infections, the clinical outcomes associated with different Rickettsia species can be radically different ranging from a rash to death. ${ }^{40}$ Furthermore, critical tick vectors often differ in habitat and seasonality, which will influence strategies for prevention and control. Only when we understand all of the aspects of a vector-borne disease can we hope to formulate effective control and management solutions.

Received February 24, 2020. Accepted for publication May 15, 2020.

Published online June 22, 2020.

Note: Supplemental table appears at www.ajtmh.org.

Acknowledgments: We are grateful to the members of the county mosquito control community in all $21 \mathrm{New}$ Jersey counties that collected most of the tick specimens examined. We are also indebted to Ju Jiang from the Henry M. Jackson Foundation for sharing unpublished SOPs for her 17-kD "all rickettsia" Taqman.
Financial support: This publication was funded by Multistate NE1943 to D. M. F. and by the Northeastern IPM Center through Grant \#201470006-22484, both from the U.S. Department of Agriculture and National Institute of Food and Agriculture to A. M. E. and D. M. F.

Authors' addresses: James Occi and Dina M. Fonseca, Department of Entomology, Center for Vector Biology, Rutgers University, New Brunswick, NJ, E-mails: james.occi@rutgers.edu and dina.fonseca@ rutgers.edu. Ashley Goncalves, University of Miami Biology Department, Coral Gables, FL, E-mail: ashmgon@gmail.com. Andrea M. Egizi, Monmouth County Mosquito Control Division, Tick-borne Diseases Laboratory, Tinton Falls, NJ, E-mail: andrea.egizi@co. monmouth.nj.us.

\section{REFERENCES}

1. Gillespie JJ et al., 2008. Rickettsia phylogenomics: unwinding the intricacies of obligate intracellular life. PLoS One 3: e2018.

2. Fournier PE, Raoult D, 2009. Current knowledge on phylogeny and taxonomy of Rickettsia spp. Ann N Y Acad Sci 1166: 1-11.

3. Gillespie JJ, Ammerman NC, Beier-Sexton M, Sobral BS, Azad AF, 2009. Louse- and flea-borne rickettsioses: biological and genomic analyses. Vet Res 40: 12.

4. Kurtti TJ, Felsheim RF, Burkhardt NY, Oliver JD, Heu CC, Munderloh UG, 2015. Rickettsia buchneri sp. nov., a rickettsial endosymbiont of the blacklegged tick Ixodes scapularis. Int J Syst Evol Microbiol 65: 965-970.

5. Heitman KN, Drexler NA, Cherry-Brown D, Peterson AE, Armstrong PA, Kersh GJ, 2019. National surveillance data show increase in spotted fever rickettsiosis: United States, 2016-2017. Am J Public Health 109: 719-721.

6. Dantas-Torres F, 2007. Rocky Mountain spotted fever. Lancet Infect Dis 7: 724-732.

7. Blanton LS, 2019. The rickettsioses: a practical update. Infect Dis Clin North Am 33: 213-229. 
8. Dahlgren FS, Paddock CD, Springer YP, Eisen RJ, Behravesh CB, 2016. Expanding range of Amblyomma americanum and simultaneous changes in the epidemiology of spotted fever group rickettsiosis in the United States. Am J Trop Med Hyg 94: 35-42.

9. Ricketts HT, 1907. Observation of the virus and means of transmission of Rocky Mountain spotted fever. $J$ Infect Dis 4: 141-153.

10. Parker RR, Philip CB, Jellison WL, 1933. Rocky Mountain spotted fever: potentialities of tick transmission in relation togeographical occurrence in the United States. Am J Trop Med 13: $341-378$

11. Burgdorfer W, 1969. Ecology of tick vectors of American spotted fever. Bull World Health Organ 40: 375-381.

12. Loving SM, Smith AB, DiSalvo AF, Burgdorfer W, 1978. Distribution and prevalence of spotted fever group Rickettsiae in ticks from South Carolina with an epidemiological survey of persons bitten by infected ticks. Am J Trop Med Hyg 27: 1255-1260.

13. Magnarelli LA, Anderson JF, Burgdorfer W, Philip RN, Chappell WA, 1985. Spotted fever group Rickettsiae in immature and adult ticks (Acari: Ixodidae) from a focus of Rocky Mountain spotted fever in Connecticut. Can J Microbiol 31: 1131-1135.

14. Kakumanu ML, Ponnusamy L, Sutton H, Meshnick SR, Nicholson WL, Apperson CS, 2018. Prevalence of Rickettsia Species (Rickettsiales: Rickettsiaceae) in Dermacentor variabilis ticks (Acari: Ixodidae) in North Carolina. J Med Entomol 55: 1284-1291.

15. Burgdorfer W, Hayes SF, Mavros AJ, 1981. Nonpathogenic Rickettsiae in Dermacentor andersoni: a limiting factor for the distribution of Rickettsia rickettsii. Burgdorfer W, Anacker RL,eds. Rickettsiae and Rickettsial Diseases. New York, NY: Academic Press, 585.

16. Macaluso KR, Sonenshine DE, Ceraul SM, Azad AF, 2002. Rickettsial infection in Dermacentor variabilis (Acari: Ixodidae) inhibits transovarial transmission of a second Rickettsia. J Med Entomol 39: 809-813.

17. Carmichael JR, Fuerst PA, 2010. Molecular detection of Rickettsia bellii, Rickettsia montanensis, and Rickettsia rickettsii in a Dermacentor variabilis tick from nature. Vector Borne Zoonotic Dis 10: 111-115.

18. Jordan RA, Egizi A, 2019. The growing importance of lone star ticks in a Lyme disease endemic county: passive tick surveillance in Monmouth county, NJ, 2006-2016. PLoS One 14: e0211778.

19. Levin ML, Zemtsova GE, Killmaster LF, Snellgrove A, Schumacher LBM, 2017. Vector competence of Amblyomma americanum (Acari: Ixodidae) for Rickettsia rickettsii. Ticks Tick Borne Dis 8: 615-622.

20. Delisle J, Mendell NL, Stull-Lane A, Bloch KC, Bouyer DH, Moncayo AC, 2016. Human infections by multiple spotted fever group Rickettsiae in Tennessee. Am J Trop Med Hyg 94: 1212-1217.

21. Goddard J, 2003. Experimental infection of lone star ticks, Amblyomma americanum (L.), with Rickettsia parkeri and exposure of guinea pigs to the agent. JMed Entomol 40:686-689.

22. Egizi A, Gable S, Jordan RA, 2020. Rickettsia spp. infecting lone star ticks (Amblyomma americanum) (Acari: Ixodidae) in Monmouth county, New Jersey. J Med Entomol 57: 974-978.

23. Maestas LP, Reeser SR, McGay PJ, Buoni MH, 2020. Surveillance for Amblyomma maculatum (Acari: Ixodidae) and Rickettsia parkeri (Rickettsiales: Rickettsiaceae) in the state of Delaware, and their public health implications. J Med Entomol 57: 979-983.

24. Paddock CD, Goddard J, 2015. The evolving medical and veterinary importance of the Gulf Coast tick (Acari: Ixodidae). J Med Entomol 52: 230-252.

25. Egizi AM, Occi JL, Price DC, Fonseca DM, 2019. Leveraging the expertise of the New Jersey mosquito control community to jump start standardized tick surveillance. Insects 10: E219.

26. Smith C, Cole MM, Gouck HK, 1946. Biology and Control of the American Dog Tick. Washington, DC: US Department of Agriculture. Bulletin\#905.
27. Smart DL, Caccamise DF, 1988. Population dynamics of the American dog tick (Acari: Ixodidae) in relation to small mammal hosts. J Med Entomol 25: 515-522.

28. Keirans JE, Litwak TR, 1989. Pictorial key to the adults of hard ticks, family Ixodidae (Ixodida: Ixodoidea), east of the Mississippi River. J Med Entomol 26: 435-448.

29. Yunker C, Kierns JE, Clifford CM, 1986. Dermacentor ticks (Acari: Ixodoiae: Ixodidae) ofthe new World: a scanning electron microscope atlas. Proc Entomol Soc Wash 88: 609-627.

30. Jiang J, Stromdahl EY, Richards AL, 2012. Detection of Rickettsia parkeri and candidatus Rickettsia andeanae in Amblyomma maculatum Gulf Coast ticks collected from humans in the United States. Vector Borne Zoonotic Dis 12: 175-182.

31. Regnery RL, Spruill CL, Plikaytis BD, 1991. Genotypic identification of Rickettsiae and estimation of intraspecies sequence divergence for portions of two rickettsial genes. J Bacteriol 173: 1576-1589.

32. Gu W, Lampman R, Novak RJ, 2003. Problems in estimating mosquito infection rates using minimum infection rate. $J$ Med Entomol 40: 595-596.

33. McQuiston JH, Zemtsova G, Perniciaro J, Hutson M, Singleton J, Nicholson WL, Levin ML, 2012. A febrile spotted fever group Rickettsia infection after a bite from a Dermacentor variabilis tick infected with Rickettsia montanensis. Vector Borne Zoonotic Dis 12: 1059-1061.

34. Hardstone Yoshimizu M, Billeter SA, 2018. Suspected and confirmed vector-borne rickettsioses of North America associated with human diseases. Trop Med Infect Dis 3: E2.

35. Apperson CS, Engber B, Nicholson WL, Mead DG, Engel J, Yabsley MJ, Dail K, Johnson J, Watson DW, 2008. Tick-borne diseases in North Carolina: is "Rickettsia amblyommii" a possible cause of rickettsiosis reported as Rocky Mountain spotted fever? Vector Borne Zoonotic Dis 8: 597-606.

36. Paddock CD et al., 2008. Rickettsia parkeri rickettsiosis and its clinical distinction from Rocky Mountain spotted fever. Clin Infect Dis 47: 1188-1196.

37. Stromdahl EY, Jiang J, Vince M, Richards AL, 2011. Infrequency of Rickettsia rickettsii in Dermacentor variabilis removed from humans, with comments on the role of other human-biting ticks associated with spotted fever group Rickettsiae in the United States. Vector Borne Zoonotic Dis 11: 969-977.

38. Eisen L, 2020. Stemming the rising tide of human-biting ticks and tickborne diseases, United States. Emerg Infect Dis 26: 641-647.

39. Wisely SM, Glass GE, 2019. Advancing the science of tick and tick-borne disease surveillance in the United States. Insects 10: E361.

40. Denison AM, Amin BD, Nicholson WL, Paddock CD, 2014. Detection of Rickettsia rickettsii, Rickettsia parkeri, and Rickettsia akari in skin biopsy specimens using a multiplex real-time polymerase chain reaction assay. Clin Infect Dis 59: 635-642.

41. Ammerman NC, Swanson KI, Anderson JM, Schwartz TR, Seaberg EC, Glass GE, Norris DE, 2004. Spotted-fever group Rickettsia in Dermacentor variabilis, Maryland. Emerg Infect Dis 10: 1478-1481.

42. Moncayo AC et al., 2010. Absence of Rickettsia rickettsii and occurrence of other spotted fever group Rickettsiae in ticks from Tennessee. Am J Trop Med Hyg 83: 653-657.

43. Fritzen CM, Huang J, Westby K, Freye JD, Dunlap B, Yabsley MJ, Schardein M, Dunn JR, Jones TF, Moncayo AC, 2011. Infection prevalences of common tick-borne pathogens in adult lone star ticks (Amblyomma americanum) and American dog ticks (Dermacentor variabilis) in Kentucky. Am J Trop Med Hyg 85: 718-723.

44. Nadolny RM, Wright CL, Sonenshine DE, Hynes WL, Gaff HD, 2014. Ticks and spotted fever group Rickettsiae of southeastern Virginia. Ticks Tick Borne Dis 5: 53-57.

45. Henning TC, Orr JM, Smith JD, Arias JR, Norris DE, 2014. Spotted fever group Rickettsiae in multiple hard tick species from Fairfax county, Virginia. Vector Borne Zoonotic Dis 14: 482-485.

46. Pagac BB, Miller MK, Mazzei MC, Nielsen DH, Jiang J, Richards AL, 2014. Rickettsia parkeri and Rickettsia montanensis, Kentucky and Tennessee, USA. Emerg Infect Dis 20: 1750-1752.

47. St John HK, Adams ML, Masuoka PM, Flyer-Adams JG, Jiang J, Rozmajzl PJ, Stromdahl EY, Richards AL, 2016. Prevalence, 
distribution, and development of an ecological Niche model of Dermacentor variabilis ticks positive for Rickettsia montanensis. Vector Borne Zoonotic Dis 16: 253-263.

48. Trout Fryxell RT, Hendricks BM, Pompo K, Mays SE, Paulsen DJ, Operario DJ, Houston AE, 2017. Investigating the adult Ixodid tick populations and their associated Anaplasma, ehrlichia, and Rickettsia bacteria at a Rocky Mountain spotted fever hotspot in western Tennessee. Vector Borne Zoonotic Dis 17: 527-538.

49. Hecht JA et al., 2019. Multistate survey of American dog ticks (Dermacentor variabilis) for Rickettsia species. Vector Borne Zoonotic Dis 19: 652-657.

50. Gaines DN et al., 2014. Ehrlichia and spotted fever group Rickettsiae surveillance in Amblyomma americanum in Virginia through use of a novel six-plex real-time PCR assay. Vector Borne Zoonotic Dis 14: 307-316.

51. Lee S, Kakumanu ML, Ponnusamy L, Vaughn M, Funkhouser S, Thornton H, Meshnick SR, Apperson CS, 2014. Prevalence of Rickettsiales in ticks removed from the skin of outdoor workers in North Carolina. Parasit Vectors 7: 607.

52. Gleim ER, Garrison LE, Vello MS, Savage MY, Lopez G, Berghaus RD, Yabsley MJ, 2016. Factors associated with tick bites and pathogen prevalence in ticks parasitizing humans in Georgia, USA. Parasit Vectors 9: 125
53. Miller MK, Jiang J, Truong M, Yarina T, Evans $H$, Christensen TP, Richards AL, 2016. Emerging tick-borne Rickettsia and ehrlichia at Joint Base Langley-Eustis, Fort Eustis, Virginia. US Army Med Dep J 3-6: 22-28.

54. Sayler K, Rowland J, Boyce C, Weeks E, 2017. Borrelia burgdorferi DNA absent, multiple Rickettsia spp. DNA present in ticks collected from a teaching forest in north central Florida. Ticks Tick Borne Dis 8: 53-59.

55. De Jesus CE, Ganser C, Kessler WH, White ZS, Bhosale CR, Glass GE, Wisely SM, 2019. A survey of tick-borne bacterial pathogens in Florida. Insects 10: 297.

56. Roux V, Fournier PE, Raoult D, 1996. Differentiation of spotted fever group Rickettsiae by sequencing and analysis of restriction fragment length polymorphism of PCR-amplified DNA of the gene encoding the protein rOmpA. J Clin Microbiol 34: 2058-2065.

57. Jiang J, Yarina T, Miller MK, Stromdahl EY, Richards AL, 2010. Molecular detection of Rickettsia amblyommii in Amblyomma americanum parasitizing humans. Vector Borne Zoonotic Dis 10: 329-340.

58. Jiang J, Blair PJ, Olson JG, Stromdahl E, 2005. Development of a duplex quantitative real-time PCR assay for the detection of tick-borne spotted fever group Rickettsiae and Rickettsia rickettsii. Int Rev Armed Forces Med Serv 78: 174-179. 